\title{
On the Aerodynamic Drag of Streamers and Flags
}

\author{
A. Carruthers* and A. Filippone ${ }^{\dagger}$ \\ UMIST \\ Dept. of Mechanical, Aerospace, Manufacturing Engineering \\ Manchester M60 1QD \\ United Kingdom
}

\begin{abstract}
Low speed drag and flutter measurements of rectangular flags and streamers are presented. The streamers have aspect-ratios between 10 and 30; the flag has an aspect-ratio 3.3. Operating Reynolds numbers are in the range $R e=2 \cdot 10^{5}$ to $2 \cdot 10^{6}$. Experiments are performed with three fabrics of different weight, bending rigidity and hysteresis. The streamers are fixed to a three-component force balance using two methods: by clamping of the luff on the support, and by a wire connecting luff and support. The parametric space studied includes wind speed, aspect-ratio, planform area, clamping method and fabric properties. Semi-empirical correlations for the time-averaged drag coefficient as a function of aspect-ratio are derived for cotton streamers. Flutter analysis shows that the oscillation of the streamers is complex, due to the effects of gravity and the turbulence created by the large amplitude oscillations. Power spectra density data are presented for some relevant cases.
\end{abstract}

${ }^{*}$ Graduate Student, AIAA Student Member

${ }^{\dagger}$ Lecturer, AIAA senior member. Corresponding author. E-mail: a.filippone@umist.ac.uk 


\section{Introduction}

This paper discusses the results of wind tunnel investigations on slender rectangular flags fixed at one end, and freely moving in the vertical direction. In the technical literature these flags are named ribbons, streamers, banners, or strips, depending on their aerodynamic applications. These applications include a number of aerodynamic decelerator systems (parachutes, cords and parts of the canopy), stabilisers for weapon systems (rocket-launched grenades), and other purposes, for example kites and tow bars for flying billboards. There is also interest in the wind loads on flag poles caused by high winds or excessively large flags, or as a source of drag (when high drag is desiderable).

Two types of attachment are possible, with streamers tied at one or both ends; in the latter case, they are known as tapes. However, only the former case is considered here. For clarity, the term "flag" is adopted in our discussion when the aspect-ratio is modest (less than 4). The term "streamer" will be used in all other cases.

For configurations where horizontal mounting is used, the gravity has a more significant effect. In fact, for a streamer attached at one end on a horizontal line the aerodynamic forces work against the gravity and create additional instabilities. Flutter analysis is difficult even in laminar flow.

Modern applications include technological materials, such as polyester, nylon, Mylar, Kevlar and others, but experimental data in this field are surprisingly scarce. Some drag data were published as early as 1930 by Fairthorne [1], in response to the need of data for advertising streamers towed by aircraft. The data reported by this author were for rectangular flags of aspect-ratio 0.5 to 4.0 and triangular flags of aspect-ratio 0.25 to 1.0; they showed that: 1.) The drag decreased with the increasing aspect-ratio; 2.) the non viscous drag component depended on the mass of the fabric. Hoerner [2], an important resource for drag characteristics in all fields of engineering, is not satisfactory on this chapter. Hoerner himself referred to the work of Fairthorne (cited above), and reported only drag data for ordinary (low aspect-ratio) flags.

More recently, Levin et al. [3] published drag data for eight different types of ma- 
terials, characterised by their weight (per unit length). The streamers were fixed at the trailing edge of a symmetric airfoil and free to move in the vertical direction. The aspect-ratios tested were in the range between 2 and 22. The results showed how the drag decreases with the increasing aspect-ratio, as a consequence of the fact that the axial force increases with the amplitude of fluctuation of the free edges. These authors also discussed dynamic tests, aimed at describing the effects of fluttering. This fluttering produced (obviously) more scattered results than static tests. This conclusion has been confirmed by the present study.

Auman \& Dahlke [4] investigated the drag of "ribbons" used for reducing the hazardous dud rate of rocket-launched grenades. Aspect-ratios tested were in the range of 4 to 75 , and speeds tested were from subsonic to supersonic (Mach number $M \sim 2$ ), although the data were not presented as a function of the Reynolds number. Some of these tests were made using a grenade gun. It was proved that for a flight vehicle the slender ribbons work as stabilisers, although several flight characteristics of the grenade were detected.

The stability problem of the flags was earlier investigated by Taneda [5], who studied the waving motion of flags at relatively low speeds $\left(R e=10^{4}-10^{5}\right)$. Oscillation modes and limits of stability were discussed, and a Strouhal number for the oscillations was derived. Taneda implied that the flutter frequency was unique, and that it increased with the wind speed, but this is unlikely to be the case. Furthermore, it was proved that the drag coefficient at a given Reynolds number depends on whether the flag is fluttering or not. The critical Reynolds number at which the flag reverts to a fluttering mode $\left(R e_{c} \sim 10^{4}\right)$ also creates a "jump" in the drag coefficient, that is justified by flow separation and by momentum transfer to the flow during oscillations. The experimental set-up of Taneda included vertical flags in a vertical wind tunnel flow, which eliminated most of the gravity effects. The drag of the flags, in fact, acts in the same direction of the weight, and in the laminar or transitional Reynolds numbers the flutter characteristics are relatively simple. 
Methods for theoretical modelling of flags and streamers are rare in the technical literature, except for some computer graphics applications, which will not be considered here. The experimental data reported by the authors cited are generally described using semi-empirical expressions. This is also the approach followed in the present study.

There exist theoretical analyses of two-dimensional flags in potential flow model, with focus on flutter, tension, modes of oscillation and stability. Sparenberg [6] modelled the waving motion of a membrane, and derived an equation for the flapping frequency, assuming a Kutta condition at the trailing edge. Moretti [7] studied the tension in fluttering flags under the assumption that the gravity has no effect. An interesting study on paper flutter was published by Watanabe et al [8], in which the full NavierStokes equations were used for a flutter simulation. These results were compared with a potential flow analysis on an oscillating thin airfoil, and showed surprising consistency between the two methods in terms of flutter modes prediction. In another study, Fitt \& Pope [9] derived a theory for the unsteady motion of two-dimensional flags in a potential flow, under the assumption of small perturbations. These authors studied the limits of stability of the flags in unsteady motion (depending on rigidity and mass), with clamped and flexible leading edges; they did not derive expressions for the drag.

Some flexible structures have been the subject of theoretical analysis by Alben et al. [10], who studied the drag reduction properties of bodies with certain flexural rigidity, including the characteristics and self-similarity solutions [11].

This paper fills in the technical literature with new data for streamers having "clamped" and "flexible" leading edges. The effects of oscillations of the streamers on the resulting drag have been investigated and are discussed. An analysis has been carried out to evaluate the aspect-ratio effects on the aerodynamic drag at constant wetted area. A broad parametric space is investigated. This includes, in particular, material properties, planform area, clamping method, and aspect-ratio.

Flapping frequency is also discussed in some relevant cases. It is proved that there is a complex spectrum of flutter frequencies, and that frequency separation is not possible. 


\section{Experimental Set-up}

A wind tunnel of working section $0.9 \times 0.9$ metres, 3.14 metres long, incorporating an externally mounted three-component force balance, was used to take the drag measurements. Twelve streamers of cotton fabric were made, with planform areas $0.025 \mathrm{~m}^{2}$, $0.05 \mathrm{~m}^{2}$, and $0.075 \mathrm{~m}^{2}$. For each of these streamers four aspect-ratios were considered: $3.3,10,20$, and 30 .

Additional tests were carried out using two other materials. These tests involved both nylon with one-side polyurethane coating and polyester with geometries $\mathrm{AR}=$ 10, $A=0.025 \mathrm{~m}^{2}$ and $\mathrm{AR}=20, A=0.05 \mathrm{~m}^{2}$, respectively. Additionally, a streamer with $\mathrm{AR}=30, A=0.075 \mathrm{~m}^{2}$ was made of polyester. The edges to all streamers were painted with a water and PVA adhesive mixture, which were subsequently burned to prevent or reduce fraying.

A third set of experiments involved two different methods to mount the streamers: clamped and free luff (the luff is edge of the streamer facing the wind). For both methods eyelets were punched in the luff corners of each streamer, and were then used to secure each mounting method. The clamped streamer configuration had each eyelet fastened directly to holes in the mounting rod (with $6 \mathrm{~mm}$ diameter), such that the luff was pulled taut. The eyelets were fastened via small pieces of flexible wire, the ends of which were taped to the upstream side of the rod to minimise any aerodynamic effect. The free streamer configuration also had the leading edge pulled taut by a thin piece of rigid wire the centre of which attached to the mounting rod via a length of $120 \mathrm{~mm}$ flexible wire. Again, the free ends of the wire once tied were taped to the mounting rod upstream side. The streamers tested in these experiments were nylon and polyester streamers of $\mathrm{AR}=10$ and area $A=0.025 \mathrm{~m}^{2}$, nylon and polyester streamers of $\mathrm{AR}=$ 20 and area $0.05 \mathrm{~m}^{2}$, and cotton and polyester streamers of $\mathrm{AR}=30$ and area $A=0.075$ $m^{2}$.

Therefore, a combination of planform areas and aspect-ratios were tested over a range of wind speeds, to produce a matrix of drag data, that have ultimately made 
possible the comparison of planform areas, aspect-ratios, materials and wind speeds.

The drag coefficient was calculated based on the planform area of each streamer. Reynolds numbers were calculated based on each streamer length and the free stream velocity. A velocity range of 6 to $18 \mathrm{~m} / \mathrm{s}$ was investigated, measured using a Pitot static probe in the wind tunnel. The probe connected to a high accuracy digital manometer which measured to $0.01 \mathrm{~mm} \mathrm{H}_{2} \mathrm{O}(0.098 \mathrm{~Pa})$.

Measurements were taken using a force balance through which a mounting rod was secured. A time series was taken for each data point, 2,048 samples over 2 seconds, and averaged to give a mean drag measurement. This voltage output was converted to a force measurement through a calibration curve, obtained by applying forces to the force balance centre. A vibration unit was connected through the balance and used immediately before readings were taken to reduce sticktion through the force balance collet. For each respective streamer setup the final results were averaged over a set of nine tests, three obtained on each of three separate days.

Table 1 reports the bending rigidity of the fabric, which is its stiffness (e.g. resistance to deformations) and the bending hysteresis (which characterises its recovery after deformation). The higher the bending hysteresis, the poorer the recovery of the fabric. Note that the weight of the cotton fabric is roughly three times the weight of polyester and $20 \%$ higher than the weight of nylon. On the other hand, the bending rigidity of nylon is nearly one order of magnitude larger than the cotton.

\begin{tabular}{|c|c|c|c|c|c|}
\hline Material & Weight & $\begin{array}{c}\text { Bending } \\
\text { Rigidity } \\
g r \mathrm{~cm}^{2} / \mathrm{cm}\end{array}$ & $\begin{array}{c}\text { Bending } \\
\text { Hysteresis } \\
g r \mathrm{~cm} / \mathrm{cm}\end{array}$ & AR & Area \\
\hline \hline & & & & $m^{2}$ \\
cotton & 177 & 0.0642 & 0.0446 & All & All \\
nylon & 140 & 0.4795 & 0.2103 & 10,20 & $0.025,0.05$ \\
polyester & 64 & 0.1313 & 0.0419 & $10,20,30$ & $0.025,0.05,0.075$ \\
\hline
\end{tabular}

Table 1. Material properties of streamers tested. The nylon fabric had a polyurethane coating on one side. 


\subsection{Damage to Streamers during Testing}

All streamers were damaged during testing to a greater or lesser extent, particularly at the highest speeds, when the streamers underwent small amplitude oscillations and high frequency oscillations. The smaller amplitude oscillations are confined to the leech as the wind speed is increased (the leech is the downstream or trailing edge of the streamer). The streamers having the lowest aspect-ratio $(\mathrm{AR}=10)$ suffered least damage with at most one or two fibres breaking loose. This minor damage had a negligible effect on the drag data and it could be ignored. The longest streamers $(\mathrm{AR}=30)$ suffered more notable fraying. In these cases up to $3 \mathrm{~mm}$ of the streamer leech became frayed, and up to $1 / 4$ of the streamer lengths edges effected with fibres breaking loose as with the lower aspect-ratio. The damage during testing was considered to be little enough that new streamers were only introduced for each of the mounting methods, and consequently only two of each of these streamers, smooth (polyester) and rough (cotton or nylon) for $\mathrm{AR}=20$ and 30 , were made.

Additional tests were carried out without a PVA/water edging, in order to determine the effects on the measurements being carried out. Over a short time interval, notable damage was caused to the streamers. When the damage was negligible, the drag results compared well to those with the mixture edging. It was concluded that the method used to seal the streamer edges left the drag characteristics unchanged.

\section{$3 \quad$ Results and Discussion}

The results are presented as a function of the Reynolds number, or the wind speed. It was chosen to use the wind speed to compare the drag coefficients of different aspectratio, as the Reynolds number effect tends to shift the data apart.

The drag coefficient was calculated by using as reference area the projected area of one side of the streamer, Fig. 1. Data presented elsewhere (for example Ref. [4]) use the total wetted area, which is twice as large (therefore the $C_{D}$ would be half as large). 
Error bars are included in all the experimental data. (For clarity, some of the error bars have been removed; error bars have been plotted either at the extreme values of the wind speed/Reynolds number, or at specified distances between data samplings, generally $5 \%$ of the graph size.) The error bars give an indication of the repeatibility of the tests and the unsteadyness of the aerodynamic forces due to flapping and selfinduced turbulence. (This aspect was not clearly emphasised in the literature that we have reviewed.)

The amplitude of the error bar was calculated from the minimum and maximum deviation with respect to the mean value of 9 records taken at each operation point. In the results discussed below, only the error bars on the $C_{D}$ were plotted, since the error bar on the Reynolds number or the wind speed was negligible. In most cases, the error bar on the $C_{D}$ of the free streamers is considerably larger than the corresponding error bar on the clamped streamer - all other parameters being the same. This is attributed to the additional degree of freedom of the streamer in the wind tunnel.

\subsection{Drag of Cotton Streamers}

Figures 2-4 show the relationship between drag and aspect-ratio for the cotton fabric over the full range of velocities tested. The trend lines through each of the three data sets are similar. The power curve trend for the largest planform area is

$$
C_{D}=0.405 A R^{-0.494}
$$

The power curve for the lowest planform area is

$$
C_{D}=0.561 A R^{-0.480}
$$

The data show that the effect of aspect-ratio is far larger that the effect of wind speed for a given planform area. It is noted, however, that at the lowest aspect-ratio ( $\mathrm{AR}=$ 3.3) there is a relatively large scatter of data for higher planform areas, Fig. 3-4. In 
particular, there is a "jump" in the drag levels of the flag with the planform area $\mathrm{A}=$ $0.075 \mathrm{~m}^{2}$, with data clustered around two main areas.

The details of the flag data are shown in Fig. 6. For the largest planform area two distinct drag levels are observed. At the low speed range, $u=6-12 \mathrm{~m} / \mathrm{s}$, the drag level is weakly dependent on the speed. At the upper end of this band, corresponding to a Reynolds number $R e \sim 7.2 \cdot 10^{5}$, a drag crisis occurs. This is attributed to a change in oscillation mode. The higher drag encountered at low speed is due to larger volume of air being disturbed in the flapping movement of the flag. These flags oscillate with high amplitude and low frequencies at low wind speeds; the amplitudes decrease and the frequencies increase with increasing wind speed.

The value of the Reynolds number $R e=7.2 \cdot 10^{5}$ is achieved at the largest speed with a flag of planform area $\mathrm{A}=0.05 \mathrm{~m}^{2}$. No clear drag crisis is evident in this case, although the data are more erratic than in other cases.

Figures 5-8 show the effects of planform area on the drag coefficient as a function of the wind speed for cotton flags and streamers. These are the data points in the graphics of Figs. 2-4.

It is observed that the smallest area produced the highest drag; the largest area produced the lowest drag. This is due to the velocity range over which the large amplitude, low frequency oscillations take place. At the lowest velocities, the smallest catch in the wind speed, thereby producing a high amplitude, lower frequency oscillation. However, the streamers having larger area are too long and heavy, and only at a higher speeds are they able to catch the wind. Each streamer area displays high amplitude, low frequency oscillations at low speed, and low amplitude, high frequency oscillations are high speed. However, the smaller streamers, being shorter and lighter, suffer a more violent oscillation all all speeds. Indeed, the side-to-side rotational movement is notably more violent for the lower areas and in relation to its width is deflected by far greater amplitudes. This results in higher drag. 


\subsection{Effects of Fabric Type}

In addition to the cotton experiments, two other fabrics were tested: nylon with polyurethane coating on one side and polyester (Table 1) for two test cases, $\{A R=10, A=$ $\left.0.025 \mathrm{~m}^{2}\right\}$ and $\left\{A R=20, A=0.05 \mathrm{~m}^{2}\right\}$.

The materials have two distinctive properties which effect the aerodynamics, surface roughness and material weight. The polyester material is the most light weight and smoothest material. Both attributes combine to result in a significantly lowered drag compared to the other two fabrics. The cotton material is notably the heaviest, and is of relatively rough weave, resulting in the highest drag characteristics.

The nylon fabric has unusual characteristics, due to the single sided coating of polyurethane which gives one side virtually as smooth as the polyester fabric, and the uncoated side virtually as rough as the cotton fabric. Combined with this is a fabric weight between the two other fabrics, which results in drag characteristics between the two. The exception to this is at high velocities, where it becomes increasingly difficult to separate the cotton and nylon streamers and indeed the nylon actually increases slightly above the drag of the cotton. This is mainly due to the bending rigidity of the nylon (Table 1), 3.5 and 7.5 times higher than cotton and polyester, respectively. Therefore, as nylon is stiffer, a drag curve which appears more constant with velocity changes is produced. At the lower velocities, when the large amplitude oscillations occur, the material is more resistant to the incident forces resulting in a lesser drag magnitude. As the velocity is increased, and the lower amplitude oscillations dominate, the streamer takes on a less violent motion, and the drag magnitude is not significantly altered.

\subsection{Effects of Mounting Method}

Figures 11-12 show the effects of mounting method on the drag characteristics of streamers of $\mathrm{AR}=20, A=0.05 \mathrm{~m}^{2}$ and $\mathrm{AR}=30, A=0.075 \mathrm{~m}^{2}$. It can be seen that as with the clamped mounting method comparison, the polyester has lower drag than both 
cotton and nylon fabrics — for both mounting methods.

The higher drag produced with the free mounting method is expected, because an increased volume of air is affected by the flow perturbation. The streamer, in fact, is allowed to move, but with relative freedom of movement at the luff. This results in increased rotational movement, as completed revolutions of the streamer are allowed. Hence a larger drag is created through this additional degree of freedom.

Thus, at lower velocities the large amplitude oscillations are given relative freedom to move, creating a more violent flow than that observed with the clamped mounting method, resulting in a widened drag deviation from the clamped mounting method. At higher velocities, whilst the streamer is pulled out flat experiencing the low amplitude oscillations, the extra freedom of movement results in a more violent motion. This results in drag magnitudes similar to each other, but slightly higher than the clamped mounting method.

The exception to this is the cotton material which exhibits little variation between mounting methods. This is most probably due to its bending rigidity, almost one order of magnitude lower than the nylon, and half the value of the polyester. This results in the different mounting method having little effect on the drag. However, nylon and polyester fabrics have a more notable difference between mounting methods, because of their significantly larger bending rigidity. This means that when the free clamping method is used the streamers have significantly more movement, an effect diminished by the low bending rigidity of the cotton.

\subsection{Modes of Oscillation}

Two flapping modes were observed. They occur along the length of the streamer and take the form of low frequency, high amplitude or high frequency, low amplitude oscillations. The two flapping motions are not mutually exclusive, but one will instead dominate, depending on the flow conditions. At low velocities the high amplitude oscillations tend to dominate; at higher velocities the lower amplitude oscillations dominate. 
A rotational movement about the streamwise axis exists for both flapping states, alternating between clockwise and anti-clockwise. The rotation gives the appearance of the streamer leech (tail end) moving in a "figure eight" pattern.

At the lower velocity range the streamers undergo a more violent motion than at the higher velocities, due to the large amplitude oscillations, which occur periodically and can be separated visually into a set cyclical motion. The streamer would begin with a standard flapping motion as outlined above. The rotational movement influence then increases so that small angles from the streamwise axis are seen in alternating positive and negative angles. As this combination of the two movements (flapping and rotational) occurs, the balance between them shifts towards the rotational movement, and larger angles followed by complete revolutions of the streamer are observed, again in both directions. When these full revolutions occur, there appears to be higher energy in the streamer, which is then lost further downstream.

The higher energy contained within the streamers means that full revolutions can appear as bursts with significantly increased deflection from the horizontal. The energy builds up from the initial two-dimensional flapping state. The two-dimensional flapping state has the lowest amplitude deflections and the lowest energy exchanged with the air stream.

From the rotational movements the cycle then reverses back to the flapping movements; this is continually repeated. The number of flaps, angled flaps and full rotations observed is dependent on mounting method, wind velocity, streamer aspect-ratio and material type.

\subsection{Flutter Frequencies}

Flutter frequencies have been measured with a hot wire placed in the wake of the streamers. The frequencies have been isolated by means of a Fast Fourier Transform. An example of power spectra density is shown in Figs. 13-15, for cotton streamers of aspect-ratios $\mathrm{AR}=10,20$ 30. The background noise is negligible in comparison with 
the output signal. The high level of turbulence behind the streamer is self induced. The main flutter frequencies are in the range of 5-30 Hz. From tests of this nature we have concluded that it is not possible to identify flutter frequencies as proposed by Taneda [5], who had the advantage of flags pulled down by the gravity in a vertical wind tunnel running at sub-critical Reynolds numbers, with fabrics up to 4 times heavier then the present tests.

Our analysis shows for the cases in Figs. 13-15 the main frequency is $13 \mathrm{~Hz}(\mathrm{AR}=$ $30), 15 \mathrm{~Hz}(\mathrm{AR}=20), 44 \mathrm{~Hz}(\mathrm{AR}=10)$. For reference, Taneda shows a flutter frequency of about $50 \mathrm{~Hz}$ for a fabric of the same weight and a flag aspect-ratio of about 1 at $u=$ $15 \mathrm{~m} / \mathrm{s}$, although the corresponding Reynolds number is one order of magnitude lower.

\section{Conclusions}

Drag measurements were taken in a low speed wind tunnel on a series of high aspectratio streamers and low aspect-ratio flags made of three different fabrics that cover a wide spectrum of weights and surface roughness. The parametric space investigated include planform areas, wind speeds and mounting methods. The characteristics can be extrapolated to fabrics of similar physical characteristics. The time-averaged drag data show that:

- Aspect-ratio effect. The drag decreases with the increasing slenderness of the streamer (all other parameters being the same), in a more marked amount the wind speed. Semi-empirical relations have been proposed to describe the set of averaged drag data.

- Planform area effect. The drag increases with the increasing planform area for all types of streamers. This effect is due to an increase of the skin friction drag more rapid than the decrease of the drag related to increased Reynolds number effects.

- Mounting method effect. This effect has to be coupled with the bending rigidity and weight of the fabric. Low bending rigidity of the cotton produces a smaller 
difference between free and clamped mounting methods. The method difference becomes very notable for nylon and polyester at low velocity.

- Fabric properties effect. The drag decreases with the weight of the fabric, and is further affected by properties such as roughness, bending rigidity and hysteresis - all other parameters being constant. It is emphasised that in the experiments described the drag is not aligned with the gravity, which affects the results, particularly at the lower speed range.

- Wind Speed $\&$ Reynolds number effect. The drag generally decreases with the increasing wind speed and Reynolds number. For cases where a critical Reynolds number is exceeded $\left(\operatorname{Re} \sim 7 \cdot 10^{5}\right)$ a drag crisis occurs.

- Flutter Modes and Frequencies. The streamers have two basic flapping motions: low amplitude - high frequency; high amplitude - low frequency oscillations. There is a rotational motion which occurs alongside both flapping motions. The flutter is generally described by a complex power spectra density, whose peak frequencies lye in the range $5-30 \mathrm{~Hz}$.

\section{Acknowledgments}

The authors thank Philip Blanco, at the Department of Physics, University of San Diego, for the many useful discussions, Dennis Cooper for his invaluable technical help in the laboratory, and Alison Harvey for the fabric testing (both at UMIST).

\section{References}

[1] RA Fairthorne. Drag of flags. Technical Report 1345, ARC Reports \& Memoranda, May 1930.

[2] S Hoerner. Fluid Dynamic Drag. published by the author, Bricktown Heights, NJ, 1965. (Chapter 3). 
[3] D Levin, G Daser, and Z Shpund. On the aerodynamic drag of ribbons. Number AIAA Paper 97-1525, 1997.

[4] LM Auman and CW Dahlke. Drag characteristics of ribbons. In AIAA Aerodyanmic Decelerator Systems Conferences, number AIAA 2001-2011, pages 131-136, Boston, MA, May 2001.

[5] S. Taneda. Waving motion of flags. J. Physical Soc. Japan, (2):392-401, Feb 1968.

[6] JA Sparenberg. On the waving motion of a flag. In Proceedings of the Koninklijke Nederlandse Akademie van Wetenschappen, Series B: Physical Sciences, volume 65, 1962.

[7] PM Moretti. Tension in fluttering flags. In 10th International Congress on Sound and Vibration, Stockholm, July 2003.

[8] Y Watanabe, K Isogai, S Suzuki, and M Sugihara. A theoretical study of paper flutter. J. Fluids \& Structures, 16(4):543-560, 2002.

[9] AD Fitt and MP Pope. The unsteady motion of two-dimensional flags with bending stiffness. J. Eng. Math, 40(3):227-248, 2001.

[10] S Alben, M Shelley, and J Zhang. Drag reduction through self-similar bending of a flexible body. Nature, 420:479-481, Dec 2002.

[11] J Zhang, S Childress, A Libchaber, and M Shelley. Flexible filaments in a flowing soap film as a model for one-dimensional flags in a two-dimensional wind. Nature, 408:835-839, 14 Dec 2000. 
$A R=3.3$

$A R=10$

$A R=20$

$A R=\mathbf{3 0}$

Figure 1: Streamers tested, showing the effect of aspect-ratio. 


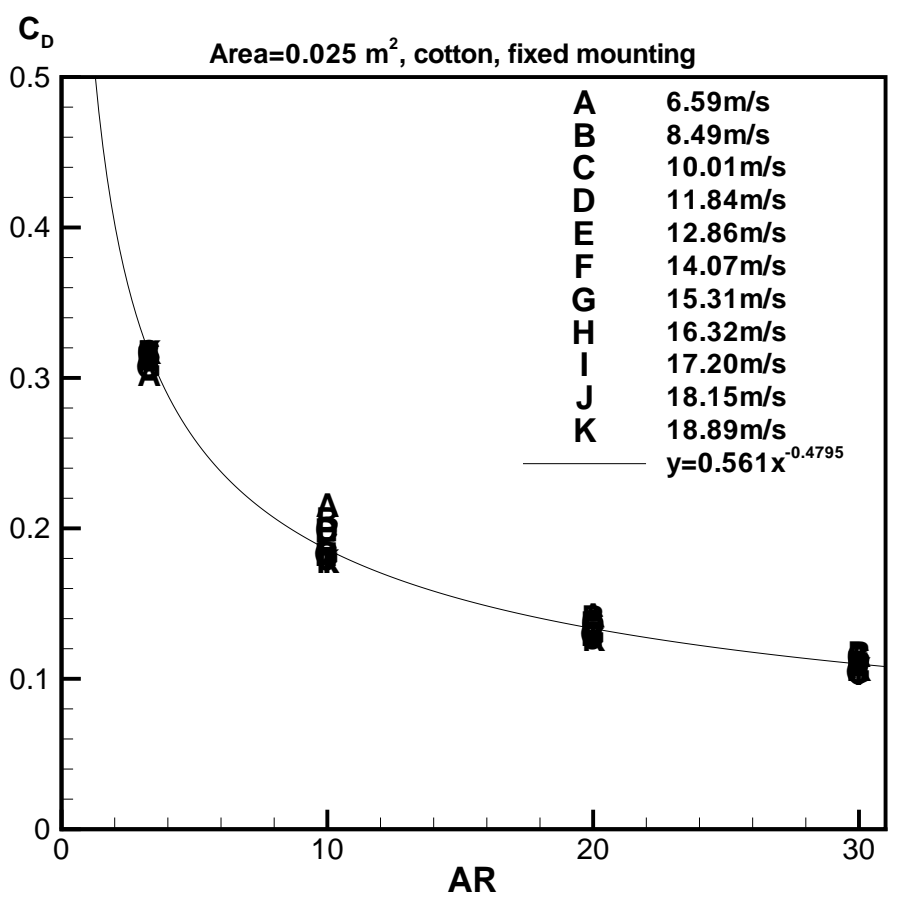

Figure 2: Drag coefficient trends at constant planform area $A=0.025 \mathrm{~m}^{2}$ for cotton streamers

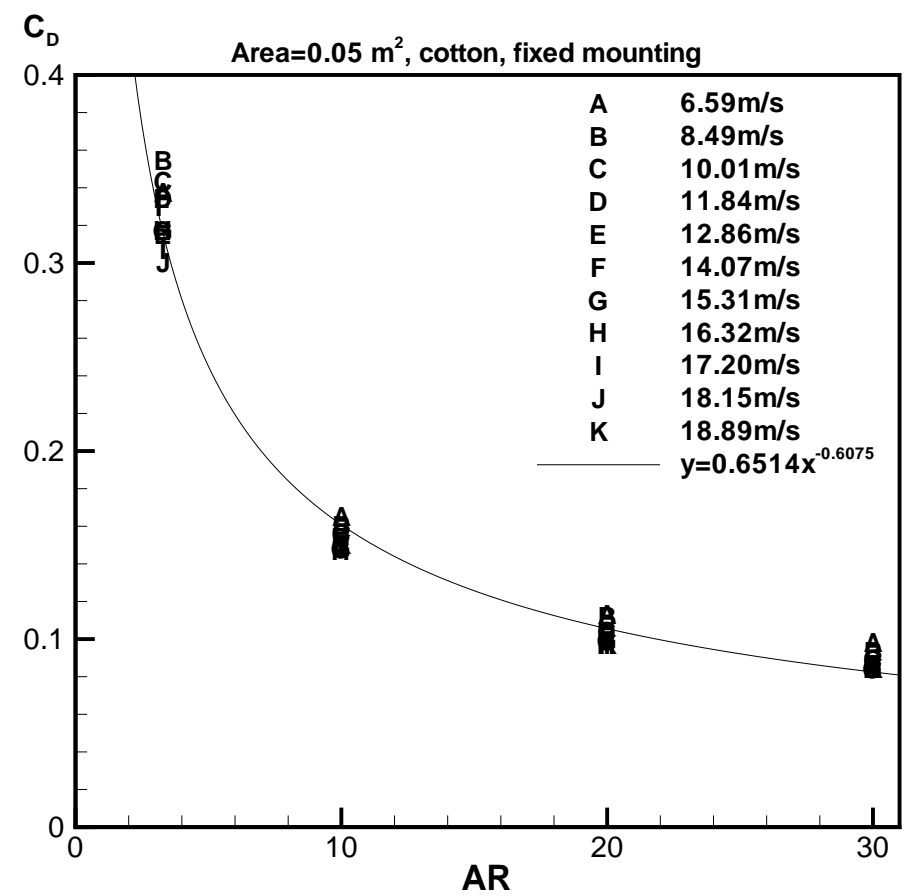

Figure 3: Drag coefficient trends at constant planform area $A=0.05 \mathrm{~m}^{2}$ for cotton streamers, and correlation with power curve. 


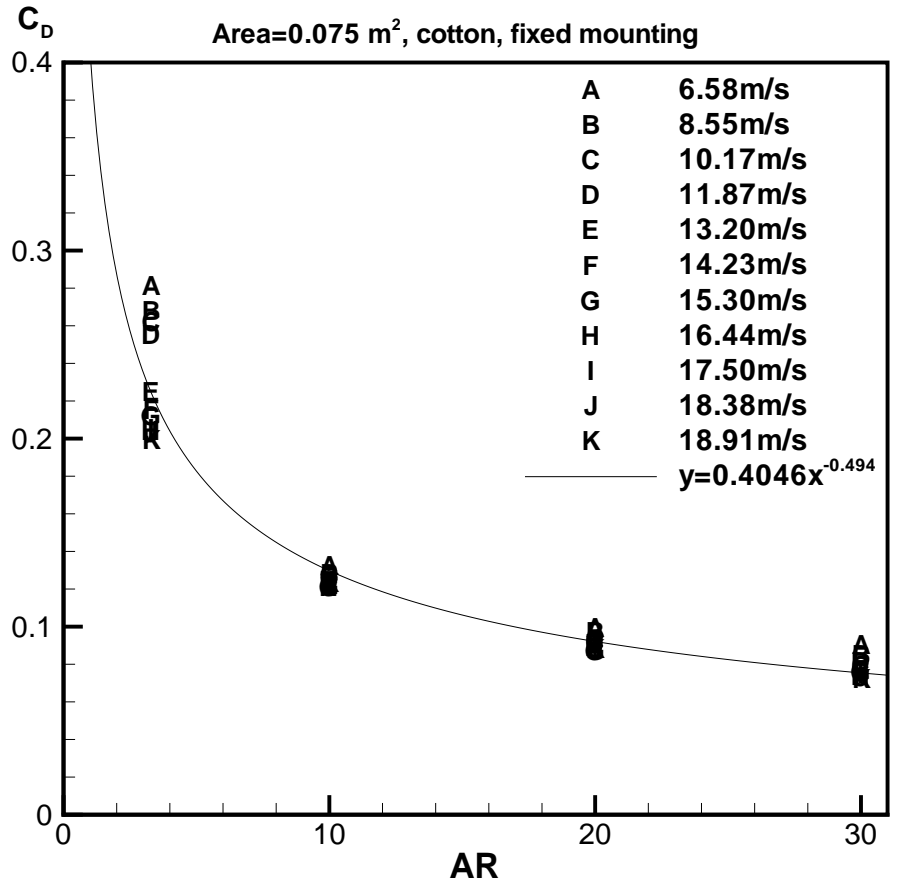

Figure 4: Drag coefficient trends at constant planform area $A=0.075 \mathrm{~m}^{2}$ for cotton streamers, and correlation with power curve. 


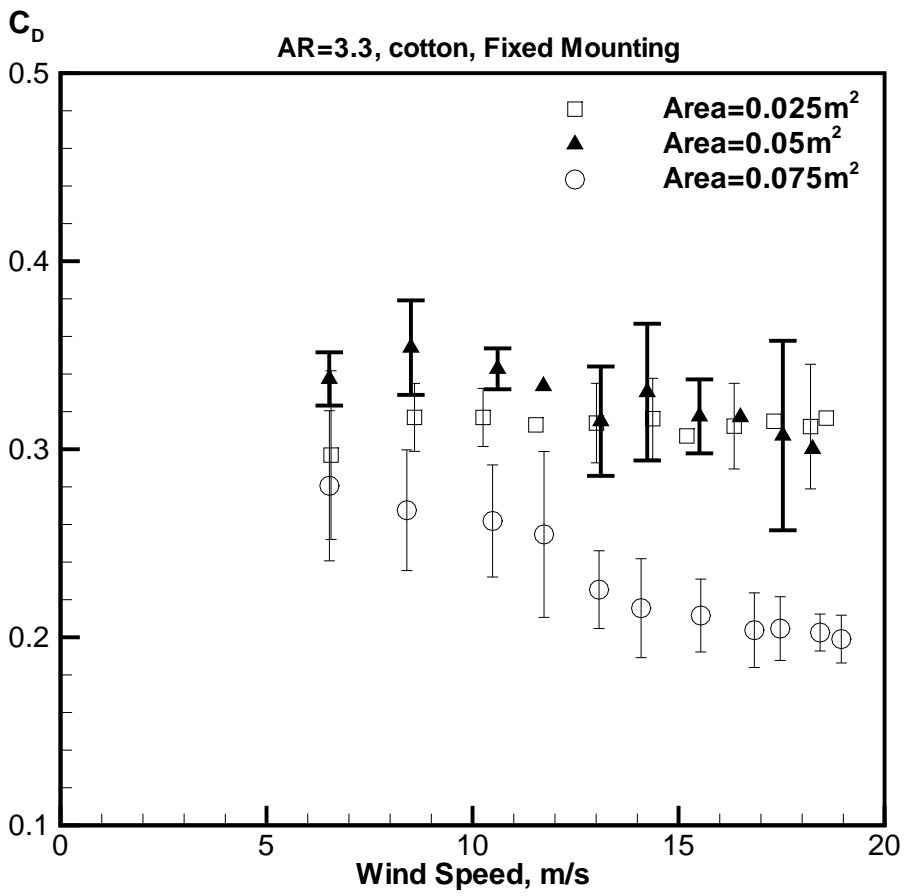

Figure 5: Effects of planform area on $C_{D}$, flag aspect-ratio $A R=3.3$.

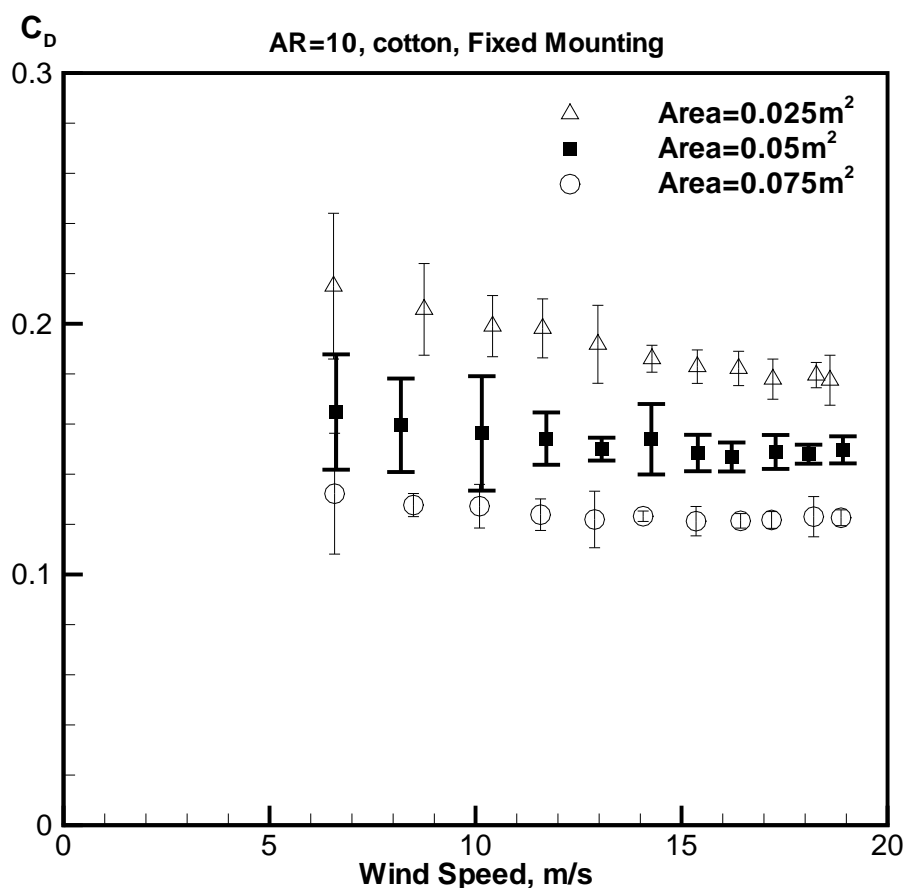

Figure 6: Effects of planform area on $C_{D}$, streamer aspect-ratio $A R=10$. 


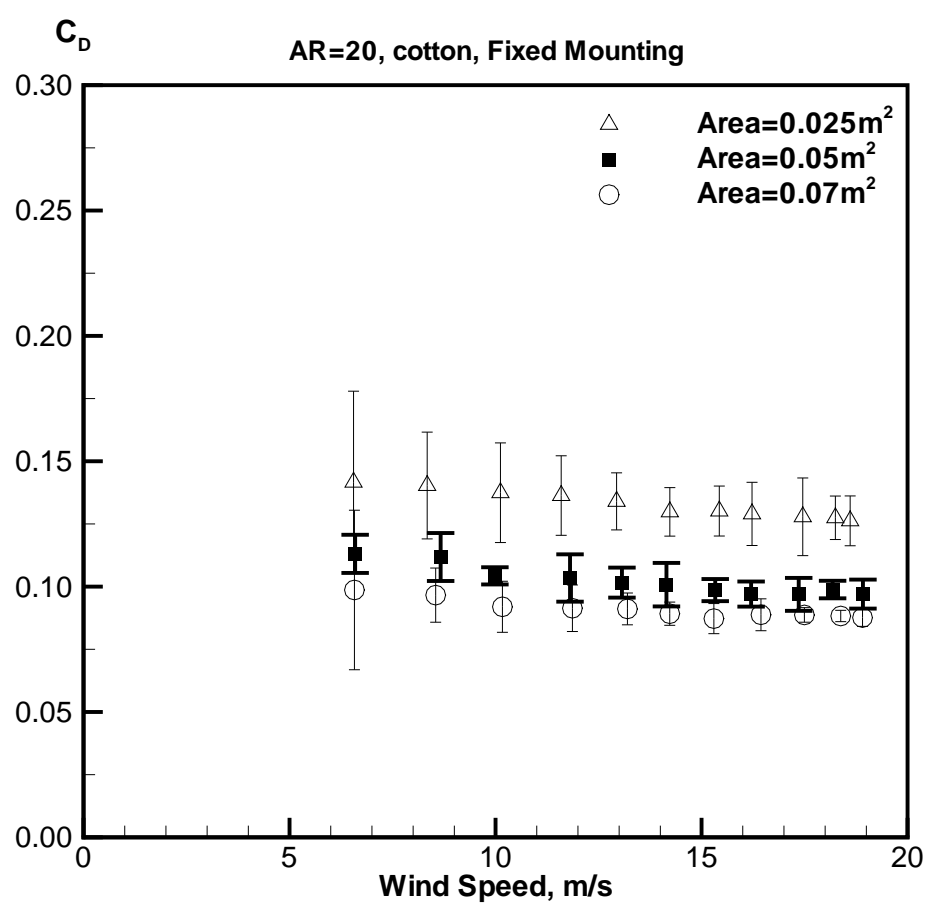

Figure 7: Effects of planform area on $C_{D}$, streamer aspect-ratio $A R=20$.

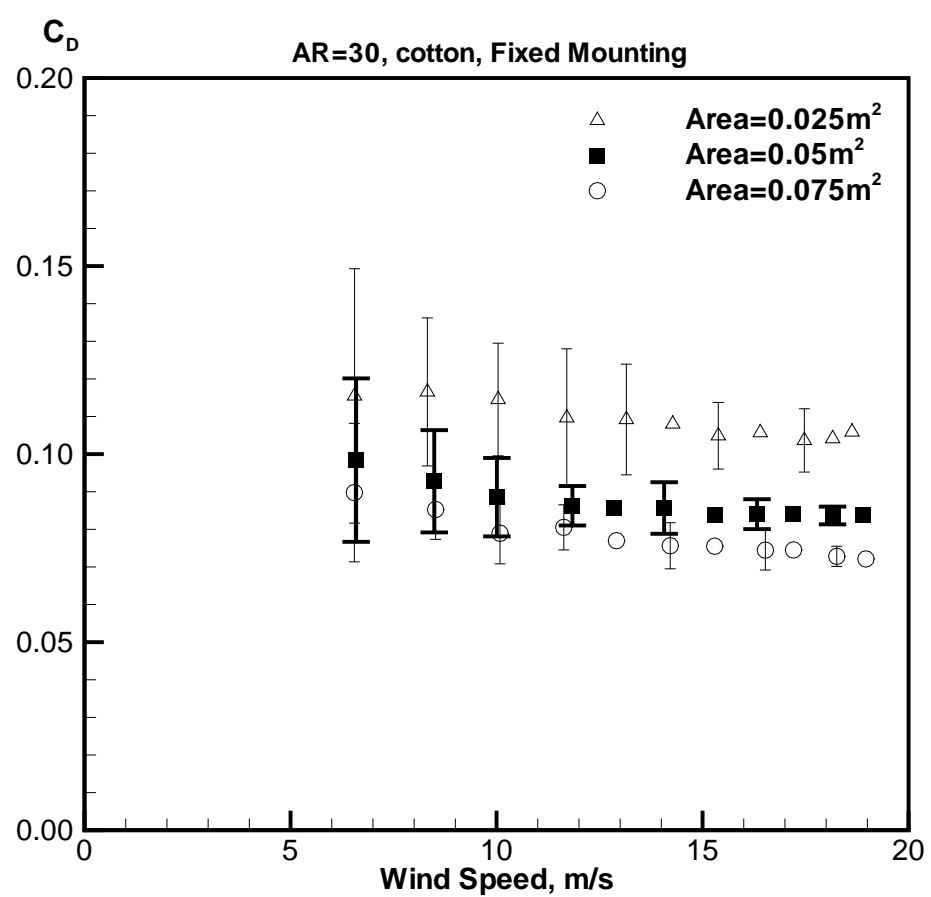

Figure 8: Effects of planform area on $C_{D}$, streamer aspect-ratio $A R=30$. 


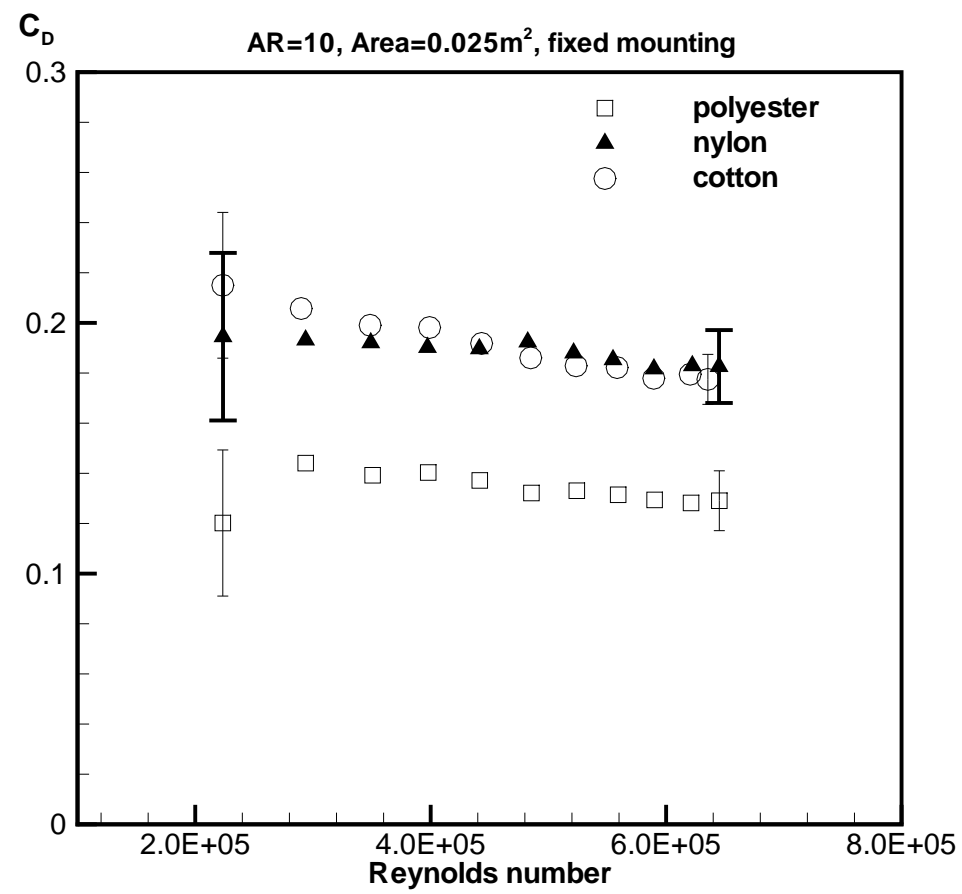

Figure 9: Effects of fabric type on $C_{D}, A R=10$.

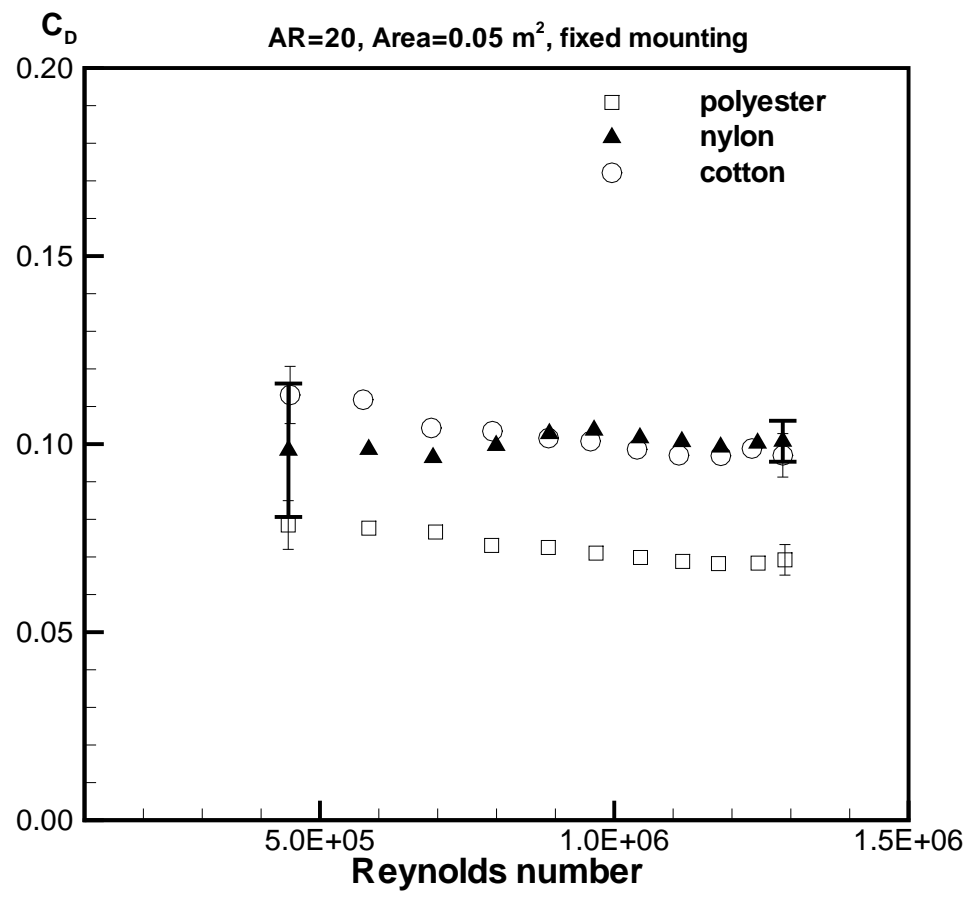

Figure 10: Effects of fabric type on $C_{D}, A R=20$. 


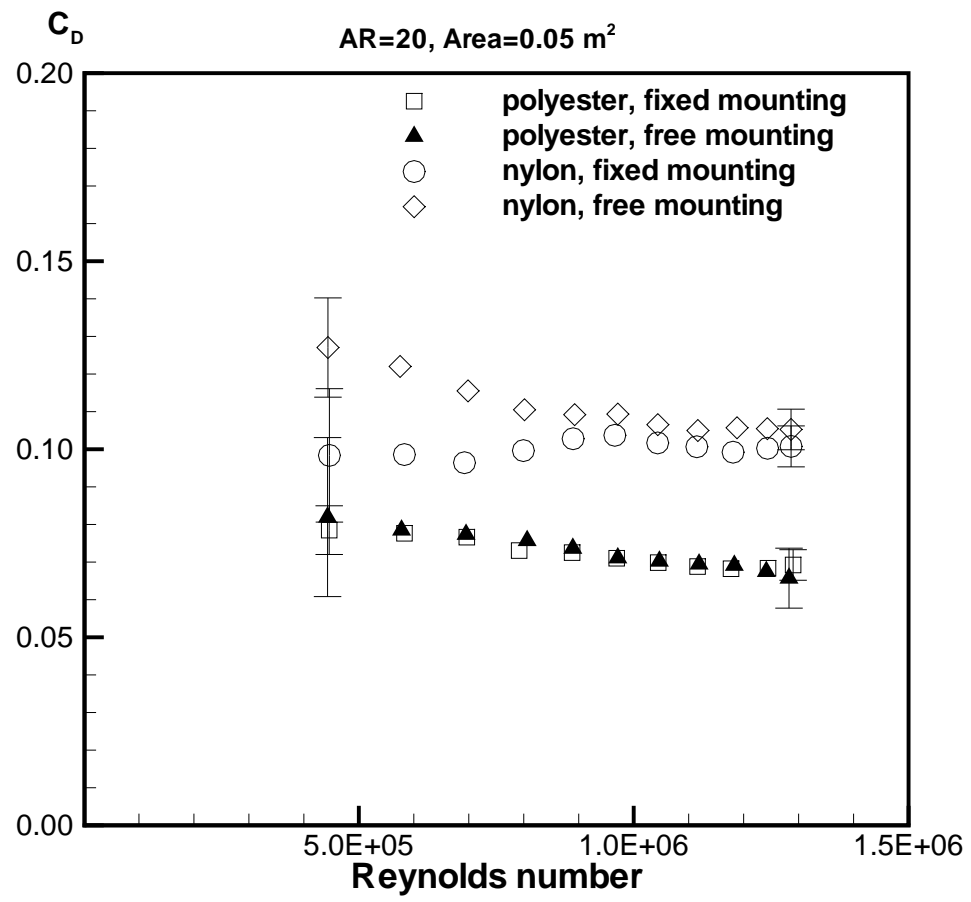

Figure 11: Effects of mounting method, $A R=20$.

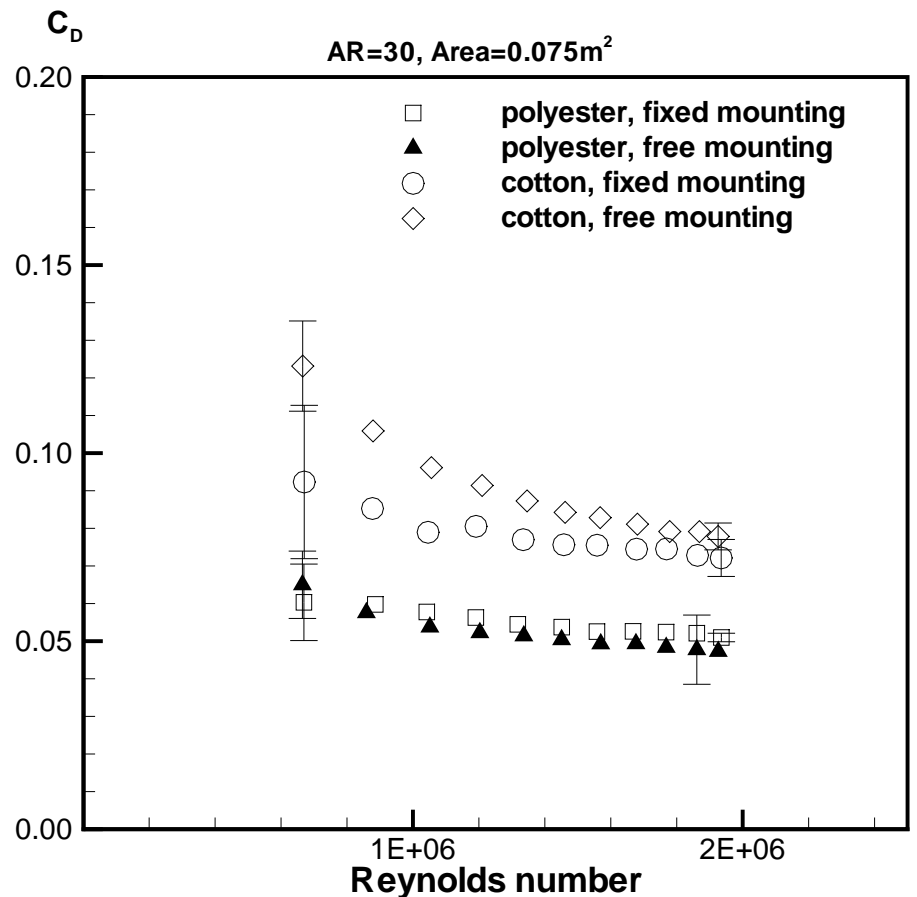

Figure 12: Effects of mounting method, $A R=30$. 


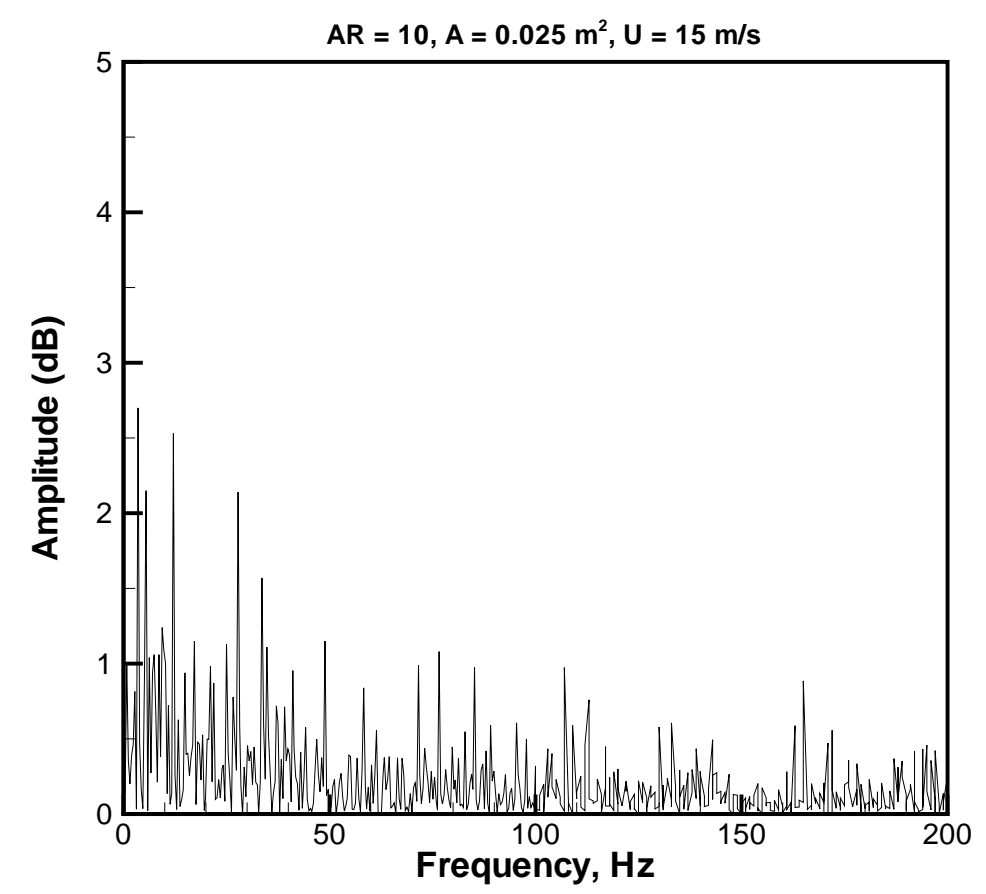

Figure 13: Power spectra density of the flutter frequency for a cotton streamer of aspect-rai"

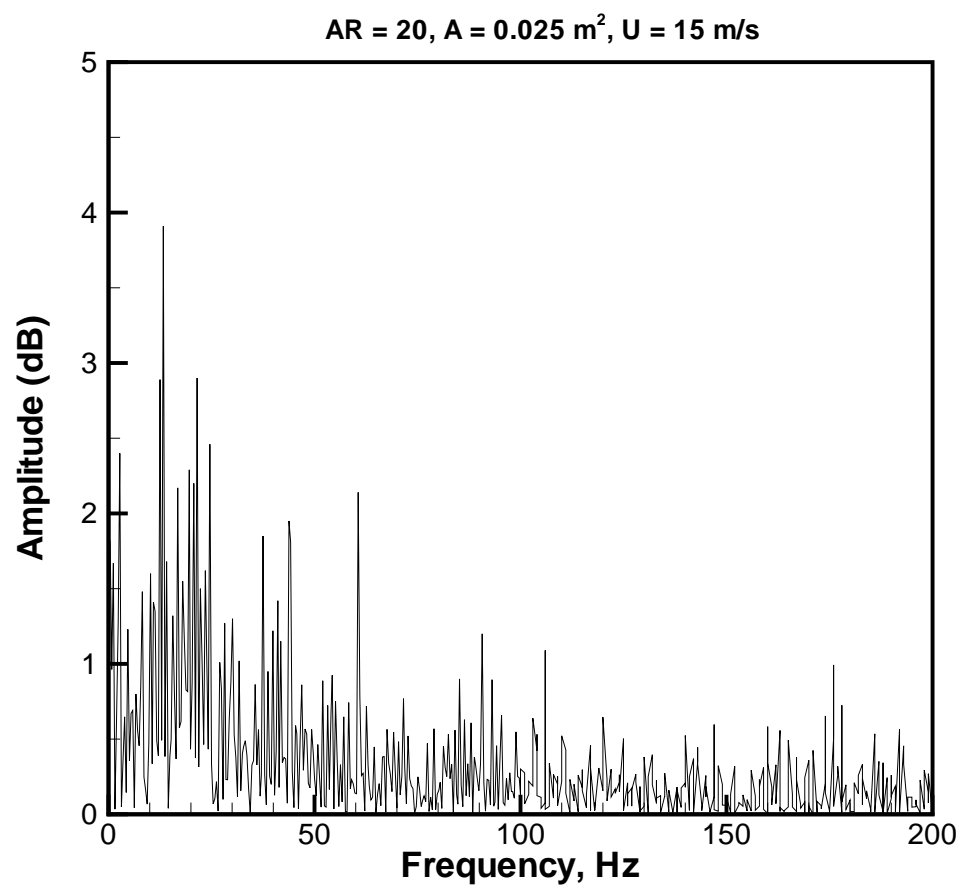

Figure 14: Power spectra density of the flutter frequency for a cotton streamer of aspect-ratio 20 . 


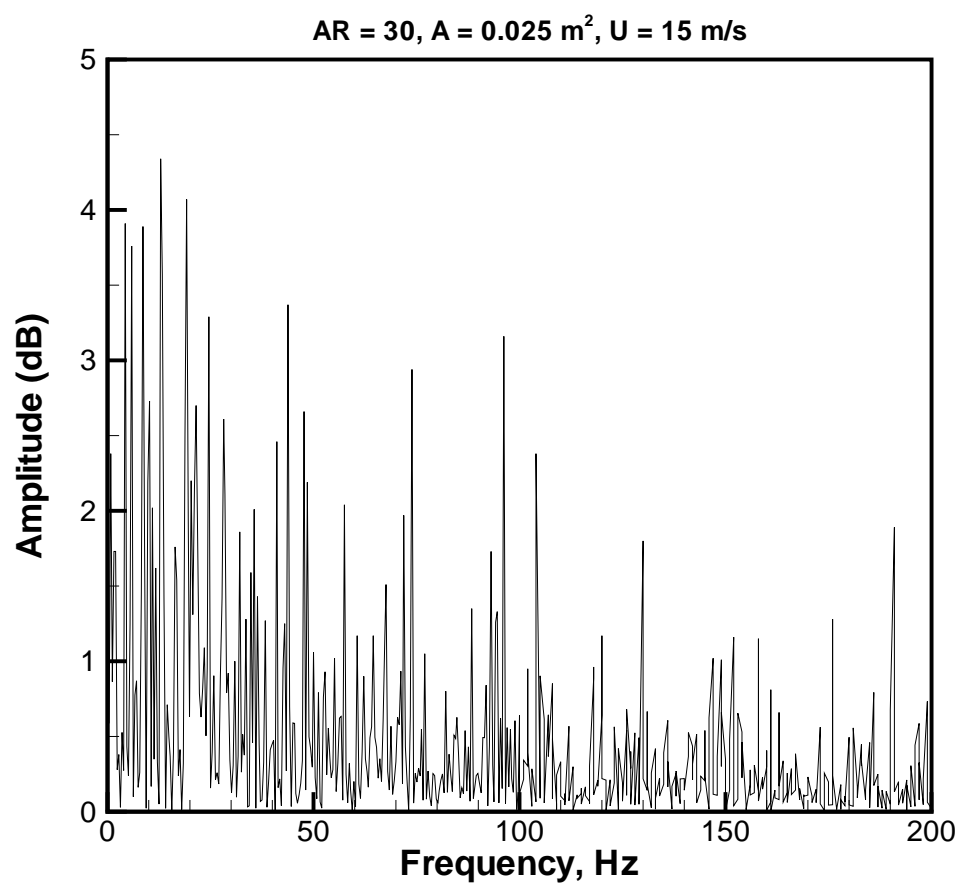

Figure 15: Power spectra density of the flutter frequency for a cotton streamer of aspect-ratio 30 . 